\title{
Promotion of GM-PHD Filtering Approach for Single-Target Tracking in Raw Data of Synthetic Aperture Radar in Spotlight Imaging Mode
}

\author{
Navid DARYASAFAR ${ }^{1}$, Ramazanali SADEGHZADEH², Mohammad NASER-MOGHADASI ${ }^{1}$ \\ ${ }^{1}$ Faculty of Engineering, Science and Research Branch, Islamic Azad University, Tehran, Iran \\ ${ }^{2}$ Faculty of Electrical Engineering, K. N. Toosi University of Technology, Tehran, Iran \\ navid_daryasafar@yahoo.com,sadeghz@eetd.kntu.ac.ir,mn.moghaddasi@srbiau.ac.ir
}

Submitted January 12, 2017 / Accepted April 9, 2017

\begin{abstract}
So far multi-antenna techniques have been used in Synthetic Aperture Radar (SAR) to track moving targets. These techniques carry out the tracking of moving targets in an imaging area, using a combination of the data received by two or several antennas. The aim of this paper is single-target tracking in SAR Spotlight imaging mode based on the promoted PHD filter. In most applications, target tracking in densely cluttered environment using radar system demands robust filtering so as to increase the tracking efficiency. Therefore, tracking of moving targets in the presence of high density clutters in environment, as the particular capability of the PHD filter, has turned it into a robust approach in SAR to track moving targets. Also as the simulation results show, using Range Cell Migration Compensation (RCMC) on SAR raw data before tracking, makes it possible to track a moving target with high quality.
\end{abstract}

\section{Keywords}

Synthetic Aperture Radar (SAR), Probability Hypothesis Density (PHD) filter, Linear Frequency Modulation (LFM), Range Cell Migration Compensation (RCMC), Random Finite Set (RFS)

\section{Introduction}

Among different target tracking methods, the concentration of this paper is on Probability Hypothesis Density (PHD) filter which spreads the first order moment of targets state Random Finite Set (RFS) or the intensity of targets state RFS in time. The RFS method considers targets as a set-valued state and also observation values as a set-valued observation; so by the viewpoint of RFS, the problem is related to the framework of Bayesian filtering. This efficient method for multi-target tracking is in fact a delicate generalization of single-target Bayesian filtering. As a matter of fact, the multi-target Bayes filter, PHD filter and also different implementation methods of it are desirable versions of filters based on RFS method [1].

In order to explain the PHD, the target state and the sensor observation models are assumed as known. In these models, $\gamma_{k}$ is the generation rate of the RFS $\Gamma_{k}$ at instance $k$ and $\Gamma_{k}$ represents RFS of spontaneous birth at time $k$; $\beta_{k \mid k-1}$ is the proliferation rate (ramification rate) of the set $\beta_{k \mid k-1}(\zeta)$ at instance $k$ for a target with a previous state of $\zeta ; p_{s, k}(\zeta)$ is the probability that a target with a previous state of $\zeta$, i.e. at instance $k-1$, also exists at instance $k$ (target survival probability); $p_{D, k}(x)$ is the probability that a target with state $x$ at instance $k$ is detected by the sensor at the same instance, and $k_{k}(\cdot)$ denotes the clutter severity of the set $k_{k}$ at instance $k$.

Using the following recurrence time equations, the PHD function is diffusible [2]:

$$
\begin{aligned}
& v_{k \mid k-1}(x)=\int p_{s, k}(\zeta) f_{k \mid k-1}(x \mid \zeta) v_{k-1}(\zeta) \mathrm{d} \zeta \\
& +\int \beta_{k \mid k-1}(x \mid \zeta) v_{k-1}(\zeta) \mathrm{d} \zeta+\gamma_{k}(x)
\end{aligned}
$$

where $f_{k \mid k-1}$ is the state transition probability density function and

$$
\begin{aligned}
& v_{k}(x)=\left[1-p_{D, K}(x)\right] v_{k \mid k-1}(x) \\
& +\sum_{z \in Z_{k}} \frac{p_{D, K}(x) g_{k}(z \mid x) v_{k \mid k-1}(x)}{K_{k}(z)+\int p_{D, K}(\zeta) g_{k}(z \mid \zeta) v_{k \mid k-1}(\zeta) \mathrm{d} \zeta} .
\end{aligned}
$$

Observations vector defined as random finite set is $\boldsymbol{Z}_{k}=\left\{\boldsymbol{z}_{k, 1}, \ldots, \boldsymbol{z}_{k, M_{k}}\right\}$, where $M_{k}$ denotes the measurement number at time $k . v_{k \mid k-1}(x)$ and $v_{k}(x)$ are predicted and updated (modified) values of the PHD function respectively.

The Sequential Monte Carlo (SMC) [3], [4] and the Gaussian Mixture (GM) [5] are the two main approaches in implementing the PHD recursion. As a large number of particles is used to approximate the multi-dimensional integrals in the SMC-PHD filter, the main drawback is its high computational burden. Moreover, to extract the target 
state estimates some clustering techniques are required, which might be often unreliable. To overcome these disadvantages, the GM-PHD filter was developed for linear Gaussian target dynamics and Gaussian birth model, in which the weights, means and covariance matrices are propagated analytically by the Kalman Filter (KF). To deal with nonlinear target dynamics and measurement models, the nonlinear Kalman filter counterparts can be directly employed. The convergence properties of two implementations were analyzed in [4], [6]. As shown in [6], the true PHD filter to any desired degree of accuracy can be approximated by the GM-PHD filter under the linear Gaussian assumption of the dynamic model. For tracking multiple maneuvering targets, similar results have been extended to handle jump Markov models [7-10]. To derive PHD smoothers, the particle and Gaussian mixture techniques have also been used [11-18]. In [19], the GM-PHD filter is extended to multi-sensor tracking system and the target state estimates are obtained sequentially at each sensor. However, the sensor registration errors are neglected.

Multi-antenna methods (hardware methods) are referred to as Interferometric Synthetic Aperture Radar (IFSAR). In these methods two or more images (complex matrix of SAR) are used to extract more information regarding target region [20], [21]. It can be noted that IFSAR system is actually an applied generalization and a different implementation of SAR systems in which independent images of a particular region are extracted using two or more imaging SAR radars. In this study, single-target tracking implementation in the received raw data by SAR is fulfilled using PHD filtering in software mode (in the MATLAB software).

\section{The Proposed Algorithm}

SAR uses an actual small scale antenna to propagate waves along the trajectory of the platform and receive the echoes of the area surface. Having received the returned echoes from the ground, a special processing begins aiming at image acquisitions from the area. The main characteristic of the hardware performance of SAR is therefore to move on the area surface, radiating and receiving waves. Having received a sufficient number of echoes, they undergo signal processing; thereby the small radar antenna behaves as a large antenna [22-26].

The impulse response of SAR, i.e., the echoes received from a spot target [27], are as follows:

$$
\begin{aligned}
& h(\tau, \eta) \approx A w_{\mathrm{r}}\left(\tau-\frac{2 R(\eta)}{c}\right) w_{\mathrm{a}}\left(\eta-\eta_{c}\right) \\
& \exp \left\{-j \frac{4 \pi f_{0} R(\eta)}{c}\right\} \exp \left\{j \pi K_{\mathrm{r}}\left(\tau-\frac{2 R(\eta)}{c}\right)^{2}\right\} .
\end{aligned}
$$

$\eta$ is the azimuth time, $A$ is the amplitude fluctuation, $w_{\mathrm{r}}(\tau-2 R(\eta) / c)$, is a range window centered on a delay of
$2 R(\eta) / c, w_{\mathrm{a}}\left(\eta-\eta_{\mathrm{c}}\right)$ is an azimuth window centered on the Doppler centroid time. $R(\eta), K_{\mathrm{r}}$ and $c$ are ranges corresponding to the azimuth time $\eta$, Chirp rate of the signal transmitted by the radar and light velocity, respectively. The principle of superposition, which is the convolution of the reflection function of the area with the SAR impulse response, can be used for the calculation of the signal received from an extensive area by SAR because of the linearity of the SAR system. After the receiver oscillator and after being transferred to base band, the signal received by SAR from an area with the reflection function $g(\tau, \eta)$ is as follows [27]:

$$
s_{\mathrm{bb}}(\tau, \eta)=g(\tau, \eta) \otimes h(\tau, \eta)+n(\tau, \eta) .
$$

$n(\tau, \eta)$ and $\otimes$ represent the additive noise in the receiver and convolution operator, respectively.

On the range-Doppler domain, applying Fourier transform on the signal received by the SAR receiver along the azimuth, it will be as follows:

$$
S_{\mathrm{rd}}\left(\tau, f_{\eta}\right) \approx w_{\mathrm{r}}\left(\tau-\frac{2 R_{\mathrm{rd}}\left(f_{\eta}\right)}{c}\right) w_{\mathrm{a}}\left(f_{\eta}-f_{\eta_{\mathrm{c}}}\right) \exp \left\{j \theta_{\mathrm{rd}}\right\} .
$$

$w_{\mathrm{r}}\left(\tau-2 R_{\mathrm{rd}}\left(f_{\eta}\right) / c\right)$ is a range window centered on a delay of $\left.2 R_{\mathrm{rd}}\left(f_{\eta}\right) / c\right)$. $\theta_{\mathrm{rd}}, R_{\mathrm{rd}}, f_{\eta}$ and $f_{\eta_{\mathrm{c}}}$ denote azimuth phase in the range Doppler domain, Range Cell Migration (RCM), azimuth frequency, and the Doppler centroid frequency, respectively. Moreover, $w_{\mathrm{a}}\left(f_{\eta}-f_{\eta_{\mathrm{c}}}\right)$ stands for azimuth window centered on the Doppler centroid frequency.

The RCM which is present in the data received by SAR for the azimuth frequency of $f_{\eta}$ is as follows [27]:

$$
R_{\mathrm{rd}}\left(f_{\eta}\right) \approx R_{0}+\frac{\lambda^{2} R_{0}}{8 V_{\mathrm{r}}^{2}} f_{\eta}^{2}
$$

$R_{0}$ and $V_{\mathrm{r}}$ show the range of closest approach and the radar velocity, respectively. Combining (5) and (6), on the range Doppler domain, the signal received by the SAR receiver is approximately as follows:

$$
\begin{aligned}
& S_{\mathrm{rd}}\left(\tau, f_{\eta}\right) \approx \\
& w_{\mathrm{r}}\left(\tau-\frac{2 R_{0}}{c}-\frac{\lambda^{2} R_{0}}{4 c V_{\mathrm{r}}^{2}} f_{\eta}^{2}\right) w_{\mathrm{a}}\left(f_{\eta}-f_{\eta_{c}}\right) \exp \left\{j \theta_{\mathrm{rd}}\right\} .
\end{aligned}
$$

The mentioned observation array, after sampling, is delivered to the SAR processor as an input signal, and as it can be seen in Fig. 1, it is processed to form radar output image on one hand and as the input to the target tracking processor, on the other hand (the proposed plan).

Now, in the tracking section of the proposed receiver, the Range Cell Migration Compensation (RCMC) process is applied to the received observation array and then the output is given to the GM-PHD algorithm to track moving targets in the area surface. Therefore it is only needed to add the RCMC approach to the PHD filtering equations 


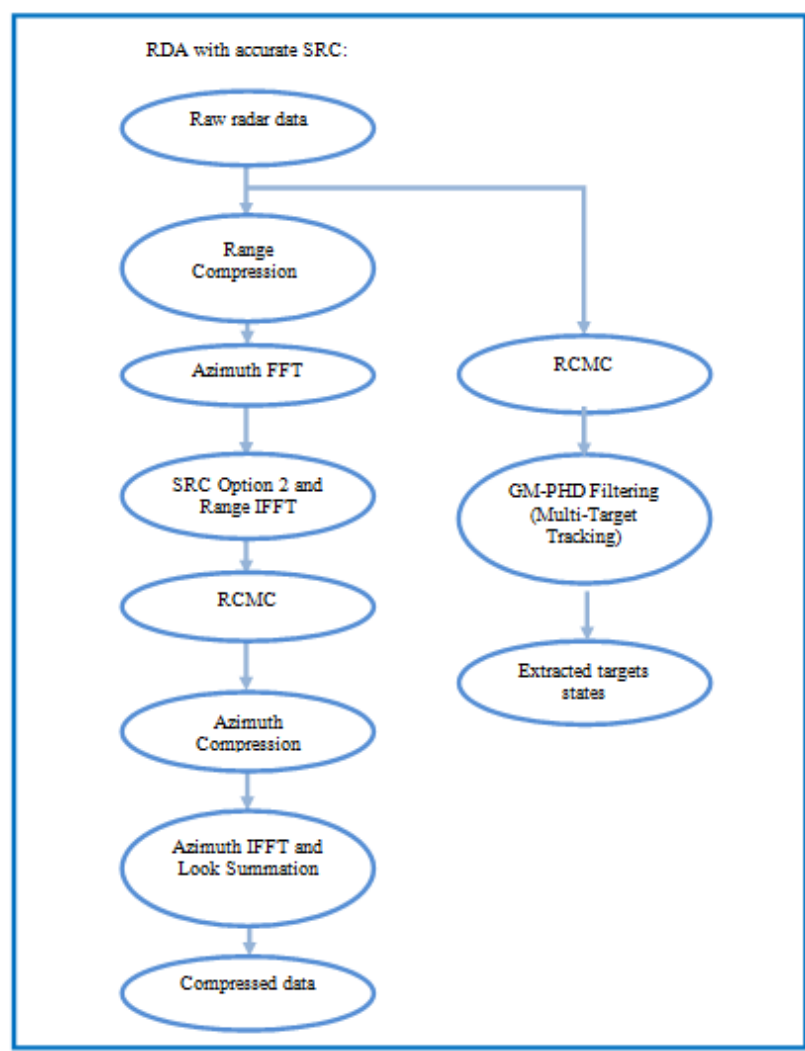

Fig. 1. The complete block diagram of the SAR processor containing the block proposed to track moving targets as well as the image formation algorithm of RangeDoppler Algorithm (RDA).

and using an additional processing of RCMC to update the sensor observation array. To this end, the $R(\cdot)$ operator is used with the following function to illustrate the RCMC operation on the observation vector received by the SAR sensor:

$$
\boldsymbol{Z}^{\mathrm{RCMC}}=R(\boldsymbol{Z})
$$

The RCM value available in the received observation vector, which is approximately as follows, should be modified from (6) as

$$
\Delta R\left(f_{\eta}\right) \approx \frac{\lambda^{2} R_{0}}{8 V_{\mathrm{r}}^{2}} f_{\eta}^{2} .
$$

$\Delta R$ indicates the proportion of RCM which needs to corrected and it is a function of the azimuth frequency $f_{\eta}$, range $R_{0}$, radar wavelength $\lambda$, and the radar platform velocity $V_{\mathrm{r}}$. After implementing the RCMC interpolation, the signal received by SAR will be as follows:

$$
\begin{aligned}
& S^{\mathrm{RCMC}}\left(\tau, f_{\eta}\right) \approx A p_{\mathrm{r}}\left(\tau-\frac{2 R_{0}}{c}\right) w_{\mathrm{a}}\left(f_{\eta}-f_{\eta_{c}}\right) \\
& \exp \left\{-\mathrm{j} \frac{4 \pi f_{0} R_{0}}{c}\right\} \exp \left\{\mathrm{j} \frac{f_{\eta}^{2}}{K_{\mathrm{a}}}\right\} .
\end{aligned}
$$

Sampling $S^{\mathrm{RCMC}}\left(\tau, f_{\eta}\right)$ on the time-range domain, the received observation vector of SAR after RCMC is ob- tained and denoted by $z^{\text {RCMC }}$. Given the observation vector $z^{\text {RCMC }}$, beginning the iteration process of the Bayesian filtering with an initial density function such as $\rho_{0}(\cdot)$, the posterior density function at the moment $k$ is tracked by the two following equations:

$$
\begin{aligned}
& p_{k \mid k-1}\left(x_{k} \mid \mathbf{z}_{1: k-1}^{\mathrm{RCMC}}\right)=\int f_{k \mid k-1}\left(x_{k} \mid x\right) p_{k-1}\left(x \mid \mathbf{z}_{1: k-1}^{\mathrm{RCMC}}\right) \mathrm{d} x, \\
& p_{k}\left(x_{k} \mid \mathbf{z}_{1: k}^{\mathrm{RCMC}}\right)=\frac{g_{k}\left(\mathbf{z}_{k}^{\mathrm{RCMC}} \mid x_{k}\right) p_{k \mid k-1}\left(x_{k} \mid \mathbf{z}_{1: k-1}^{\mathrm{RCMC}}\right)}{\int g_{k}\left(\mathbf{z}_{k}^{\mathrm{RCMC}} \mid x\right) p_{k \mid k-1}\left(x \mid \mathbf{z}_{1: k-1}^{\mathrm{RCMC}}\right) \mathrm{d} x} .
\end{aligned}
$$

Knowing the state $x_{k}$ at time $k$, probability density of receiving observation $\boldsymbol{z}_{k} \in \boldsymbol{Z}$ is $g_{k}\left(z_{k} \mid x_{k}\right)$ and, knowing all the observations $\boldsymbol{z}_{1: k}=\left(\boldsymbol{z}_{1}, \ldots, \boldsymbol{z}_{k}\right)$ up to time $k$, the probability density of state $x_{k}$ at time $k$ is given by $p_{k}\left(x_{k} \mid z_{1: k}\right)$.

The above filtering can be implemented approximately with an acceptable accuracy utilizing GM-PHD filter equations. It is therefore enough to update the GM-PHD equations obtained in [5] with $\boldsymbol{Z}^{\text {RCMC }}$ instead of Z.

After RCMC approach on received observation by receiver sensor SAR, filter prediction and update relations of GM-PHD in tracking process of SAR is followed as below:

Assume posterior intensity function at instant $k-1$ is in the form of Gaussian mixture as:

$$
v_{k \mid k-1}(x)=\sum_{i=1}^{J_{k-1}} \omega_{k-1}^{(i)} \mathrm{N}\left(x ; m_{k-1}^{(i)}, P_{k-1}^{(i)}\right)
$$

where $\mathrm{N}(. ; m, P)$ and $\omega$ denote a Gaussian density (with mean $m$ and covariance $P$ ) and weight coefficients, respectively. $J_{k-1}$ is the number of Gaussian components.

Thus, predicted intensity function at instant $k$ is in the form of Gaussian mixture as:

$$
v_{k \mid k-1}(x)=v_{S, k \mid k-1}(x)+v_{\beta, k \mid k-1}(x)+\gamma_{k}(x)
$$

where $\gamma_{k}(x)$ is also represented by (15):

$$
\begin{gathered}
\gamma_{k}(x)=\sum_{i=1}^{J_{\gamma, k}} \omega_{\gamma, k}^{(i)} \mathrm{N}\left(x ; m_{\gamma, k}^{(i)}, P_{\gamma, k}^{(i)}\right), \\
v_{S, k \mid k-1}(x)=p_{S, k} \sum_{j=1}^{J_{k-1}} \omega_{k-1}^{(j)} \mathrm{N}\left(x ; m_{S, K \mid k-1}^{(j)}, P_{S, k \mid k-1}^{(j)}\right), \\
m_{S, k \mid k-1}^{(j)}=\boldsymbol{F}_{k-1} m_{k-1}^{(j)}, \\
P_{S, k \mid k-1}^{(j)}=Q_{k-1}+\boldsymbol{F}_{k-1} P_{k-1}^{(j)} \boldsymbol{F}_{k-1}^{\mathrm{T}},
\end{gathered}
$$

in which $\boldsymbol{F}_{k-1}$ and $Q_{k-1}$ are the state transition matrix and the process noise covariance, respectively.

$$
v_{\beta, k \mid k-1}(x)=\sum_{j=1}^{J_{k-1}} \sum_{\ell=1}^{J_{\beta, k}} \omega_{k-1}^{(j)} \omega_{\beta, k}^{(\ell)} \mathrm{N}\left(x ; m_{\beta, k \mid k-1}^{(j, \ell)}, P_{\beta, k \mid k-1}^{(j, \ell)}\right),
$$




$$
\begin{gathered}
m_{\beta, k \mid k-1}^{(j, \ell)}=\boldsymbol{F}_{\beta, k-1}^{(\ell)} m_{k-1}^{(j)}+d_{\beta, k-1}^{(\ell)}, \\
P_{\beta, k \mid k-1}^{(j, \ell)}=Q_{\beta, k-1}^{(\ell)}+\boldsymbol{F}_{\beta, k-1}^{(\ell)} P_{\beta, k-1}^{(j)}\left(\boldsymbol{F}_{\beta, k-1}^{(\ell)}\right)^{\mathrm{T}} .
\end{gathered}
$$

Assume that the predicted intensity is a Gaussian mixture of the following form at time $k$ given time $k-1$ :

$$
v_{K \mid K-1}(x)=\sum_{i=1}^{J_{k \mid k-1}} \omega_{k \mid k-1}^{(i)} N\left(x ; m_{k \mid k-1}^{(i)}, P_{k \mid k-1}^{(i)}\right) \text {. }
$$

Then, the posterior intensity is also a Gaussian mixture at time $k$, and is given by:

$$
\begin{aligned}
& v_{K}(x)= \\
& \left(1-p_{D, k}\right) v_{K \mid K-1}(x)+\sum_{z^{\mathrm{RCMC}} \in Z^{\mathrm{RCMC}}} v_{D, k}\left(x ; z^{\mathrm{RCMC}}\right)
\end{aligned}
$$

where

$$
\begin{gathered}
v_{D, k}\left(x ; z^{\mathrm{RCMC}}\right)=\sum_{j=1}^{J_{k \mid k-1}} \omega_{k}^{(j)}\left(z^{\mathrm{RCMC}}\right) N\left(x ; m_{k \mid k}^{(j)}\left(z^{\mathrm{RCMC}}\right), P_{k \mid k}^{(j)}\right), \\
\omega_{k}^{(j)}\left(z^{\mathrm{RCMC}}\right)=\frac{p_{D, k} \omega_{k \mid k-1}^{(j)} q_{k}^{(j)}\left(\boldsymbol{z}^{\mathrm{RCMC}}\right)}{K_{k}\left(\boldsymbol{z}^{\mathrm{RCMC}}\right)+p_{D, k} \sum_{\ell=1}^{J_{k \mid k-1}} \omega_{k \mid k-1}^{(\ell)} q_{k}^{(\ell)}\left(\boldsymbol{z}^{\mathrm{RCMC}}\right)} \\
q_{k}^{(j)}\left(\boldsymbol{z}^{\mathrm{RCMC}}\right)=N\left(\boldsymbol{z}^{\mathrm{RCMC}} ; \boldsymbol{H}_{k} m_{k \mid k-1}^{(j)}, R_{k}+\boldsymbol{H}_{k} P_{k \mid k-1}^{(j)} \boldsymbol{H}_{k}^{\mathrm{T}}\right) \\
m_{k \mid k}^{(j)}\left(\boldsymbol{z}^{\mathrm{RCMC}}\right)=m_{k \mid k-1}^{(j)}+K_{k}^{(j)}\left(\boldsymbol{z}^{\mathrm{RCMC}}-\boldsymbol{H}_{k} m_{k \mid k-1}^{(j)}\right) \\
P_{k \mid k}^{(j)}=\left[I-K_{k}^{(j)} \boldsymbol{H}_{k}\right] P_{k \mid k-1}^{(j)}, \\
K_{k}^{(j)}=P_{k \mid k-1}^{(j)} \boldsymbol{H}_{k}^{T}\left(\boldsymbol{H}_{k} P_{k \mid k-1}^{(j)} \boldsymbol{H}_{k}^{\mathrm{T}}+R_{k}\right)^{-1}
\end{gathered}
$$

in which $\boldsymbol{H}_{k}$ and $R_{k}$ are the observation matrix and the observation noise covariance, respectively.

In the next section, simulation results of the aforementioned cases will be presented.

\section{Simulation of the Single-Target Tracking}

Owing to the SAR platform movements, the velocity data of moving targets across the region are changed and transformed in the received observation array. As a result, it should be modified and the SAR movement effect should be compensated before the implementation of the tracking process by the PHD filter. This compensation process of the received observation array (algorithm input) which is added to the PHD filtering algorithm is the same as the RCMC approach in all the SAR image formation algorithms except that it is applied to different data.

The outcome of the convolution of the signal sent by the radar and the impulse response of the SAR is equivalent to the echo received by the SAR receiver (without the noise and clutter though). Clutter which has always a Pois- son distribution in the time domain and a uniform distribution in the spatial domain [28], [29] (at any point in time) is generated accordingly and added to the echo received by the radar (the convolution of the chirp signal sent and the impulse response). After that, the observation array received by the sensor (the tracking algorithm input) is formed.

Care must be taken that fixed targets play the role of clutters and therefore considered undesirable targets. Furthermore, the main feature of Kalman and PHD filters is their ability to eliminate clutters, which was the impetus for applying the PHD filter in this paper to track moving targets in the SAR data. It should be noted that given the idea of moving targets detection and tracking, the SAR data are considered a type of radar data with high levels of clutters. The use of these filters is therefore regarded as a desirable approach for the detection and tracking of moving targets. It should however be noted that in the presence of multiple targets, the use of the Kalman filter necessitates applying techniques dedicated to data association of various targets and applying each set of data on a separate filter. The PHD filter does not face this restriction, but its only problem is the processing of actual high volume SAR data, which needs very powerful processors. The main problem in multi-target tracking is the association of data to targets, that means which observation is related to which target and this will lead to an increase in calculation load and the processing of tracking process. PHD filter operates on a single-target space and it has not the data association complexities. In fact, PHD filter transforms multi-target tracking into a single-target space and implements tracking process. Data association algorithms are often divided into Bayesian and non-Bayesian approaches. Non-Bayesian approaches utilize an optimization algorithm to find a solution for data association. On the other hand, Bayesian algorithms employ statistical theory for data association and conclude techniques such as Maximum A Posteriori (MAP).

In these simulations, the space borne Synthetic Aperture Radar (SAR) is in the spotlight imaging mode. The values of the radar's main parameters are presented in Tab. 1.

\begin{tabular}{|c|c|c|}
\hline Parameter & Description & Value \\
\hline$R_{0}$ & radar near range $(\mathrm{km})$ & 825 \\
\hline$V_{\mathrm{r}}$ & $\begin{array}{c}\text { velocity of platform } \\
(\mathrm{m} / \mathrm{s})\end{array}$ & 7619 \\
\hline$\rho_{\mathrm{r}}$ & $\begin{array}{c}\text { radar range resolution } \\
(\mathrm{m})\end{array}$ & 0.6 \\
\hline$V_{\mathrm{t}}$ & $\begin{array}{c}\text { velocity of targets } \\
(\mathrm{m} / \mathrm{s})\end{array}$ & $20-60$ \\
\hline PRF & $\begin{array}{c}\text { pulse repetition } \\
\text { frequency }(\mathrm{kHz})\end{array}$ & 3 \\
\hline$f_{\mathrm{S}}$ & sampling rate $(\mathrm{MHz})$ & 187.5 \\
\hline$f_{\mathrm{c}}$ & $\begin{array}{c}\text { radar center frequency } \\
(\mathrm{GHz})\end{array}$ & 9.6 \\
\hline Chirp_BW & $\begin{array}{c}\text { transmitted chirp } \\
\text { bandwidth }(\mathrm{Hz})\end{array}$ & $2.5415 \mathrm{e}+08$ \\
\hline$T_{\mathrm{r}}$ & pulse width $(\mathrm{sec})$ & $8.0000 \mathrm{e}-05$ \\
\hline
\end{tabular}

Tab. 1. Simulation parameters. 
A moving target with the trajectory shown in Fig. 2 is considered as an example.

For better illustration of the tracking performance over time, the target movement and also the sensor observations are shown along the perpendicular axes $\mathrm{X}$ and $\mathrm{Y}$ separately over time. Figure 3 shows the observation values of the targets and clutters along the perpendicular axes separately over time. The parameter N_Clutter represents the mean number of the Poisson process of generating clutter at every instance of time, which is equal to 20 in Fig. 3.

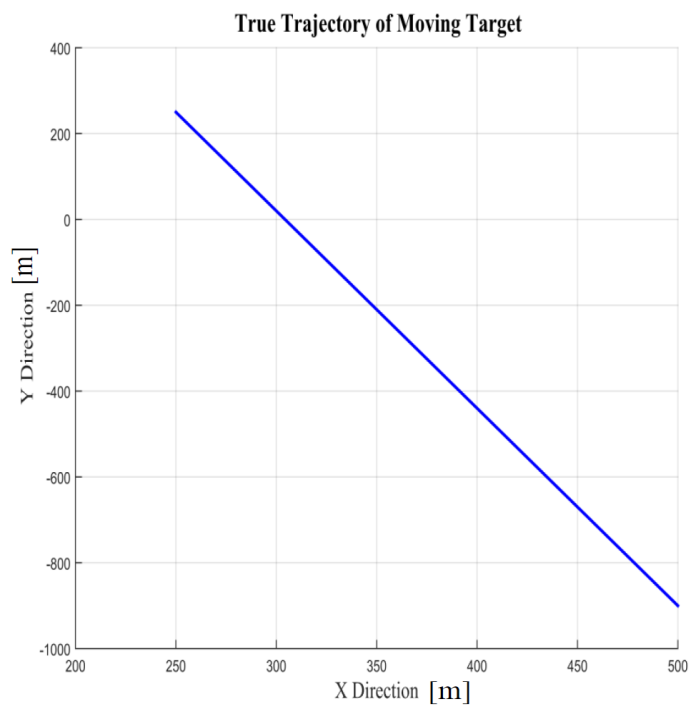

Fig. 2. A sample trajectory of a moving target in the single target scenario.
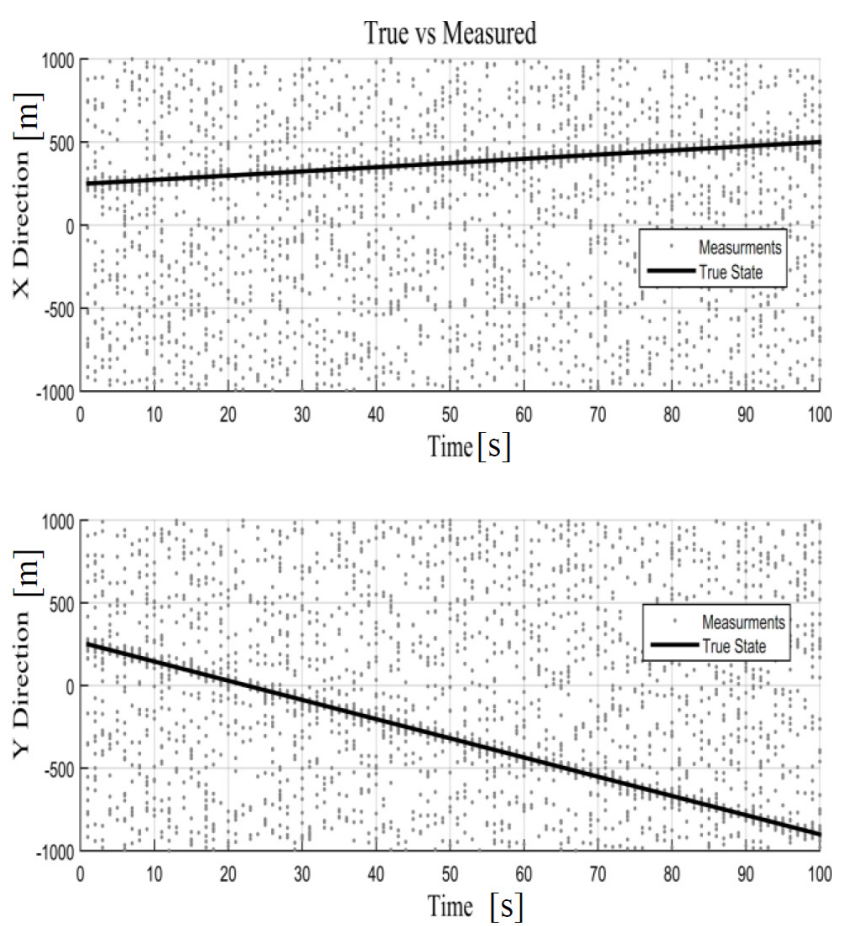

Fig. 3. Observation values of the target and clutter by the sensor along the perpendicular axes $\mathrm{X}$ and $\mathrm{Y}$ separately over time for the single target shown in Fig. 2, N_Clutter $=20$ and after RCMC.
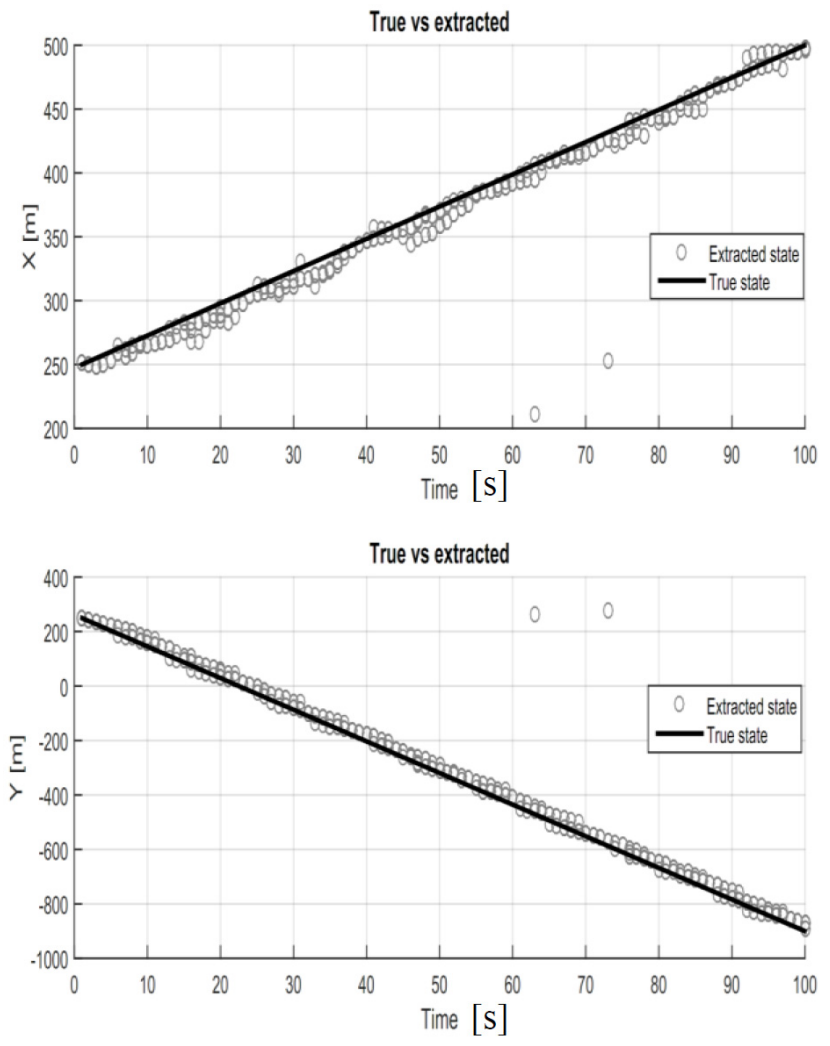

Fig. 4. State estimation of the target using the promoted GMPHD algorithm along the perpendicular axes $\mathrm{X}$ and $\mathrm{Y}$ separately over time for the single target shown in Fig. 2 and $\mathrm{N}$ Clutter $=20$.

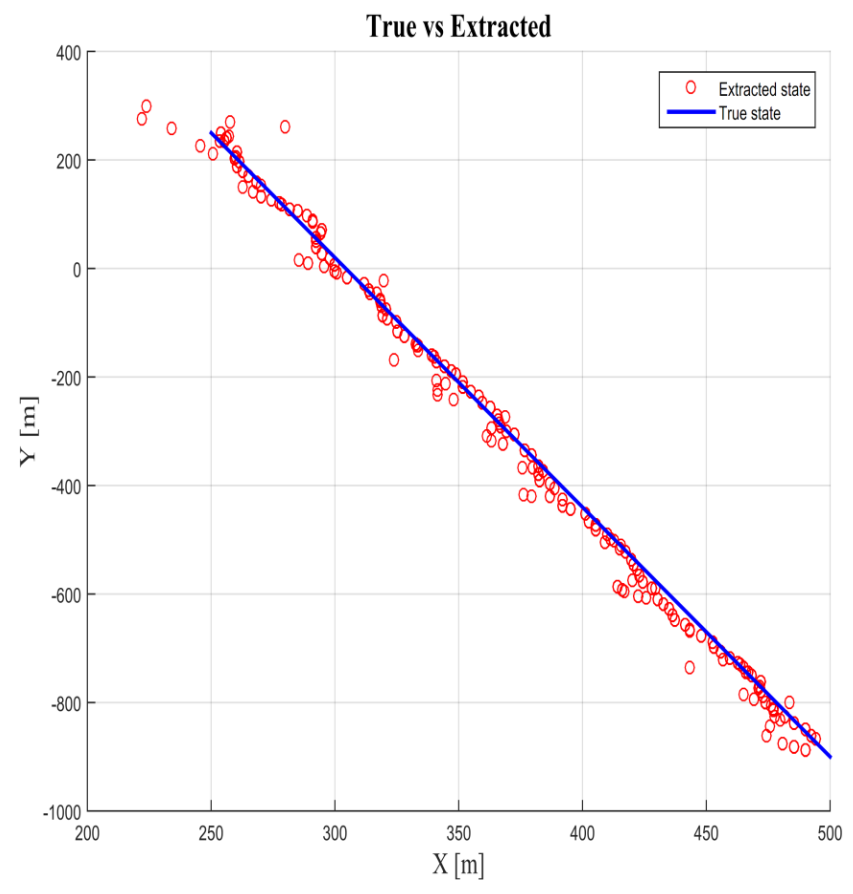

Fig. 5. The 2D target trajectory and the output of the tracking approach by the use of the promoted GM-PHD algorithm for the single target shown in Fig. 2 and N_Clutter $=20$.

An important parameter for the quality assessment of the tracking process suggested is $\mathrm{N}$ Clutter. It means that it is expected that the tracking approach performs well for 
different and particularly high values of the parameter. The output of the proposed approach (the target state at every moment) for the same target shown in Fig. 2 is exhibited in Fig. 4. The continuous curve in Fig. 4 represents the true state (position) of the target, while circles denote the target state at any instance extracted by the promoted GM-PHD algorithm.

The 2D target trajectory as well as the tracking result obtained by the promoted GM-PHD algorithm is shown in Fig. 5.

Moreover, in order to ensure that the proposed tracking plan is robust in the presence of highly dense clutter, the recent simulations have been repeated for N_Clutter $=200$, which are all shown in Figs. 6-8.

The $2 \mathrm{D}$ target trajectory as well as the tracking result obtained by the promoted GM-PHD algorithm is shown in Fig. $8($ N_Clutter $=200)$.
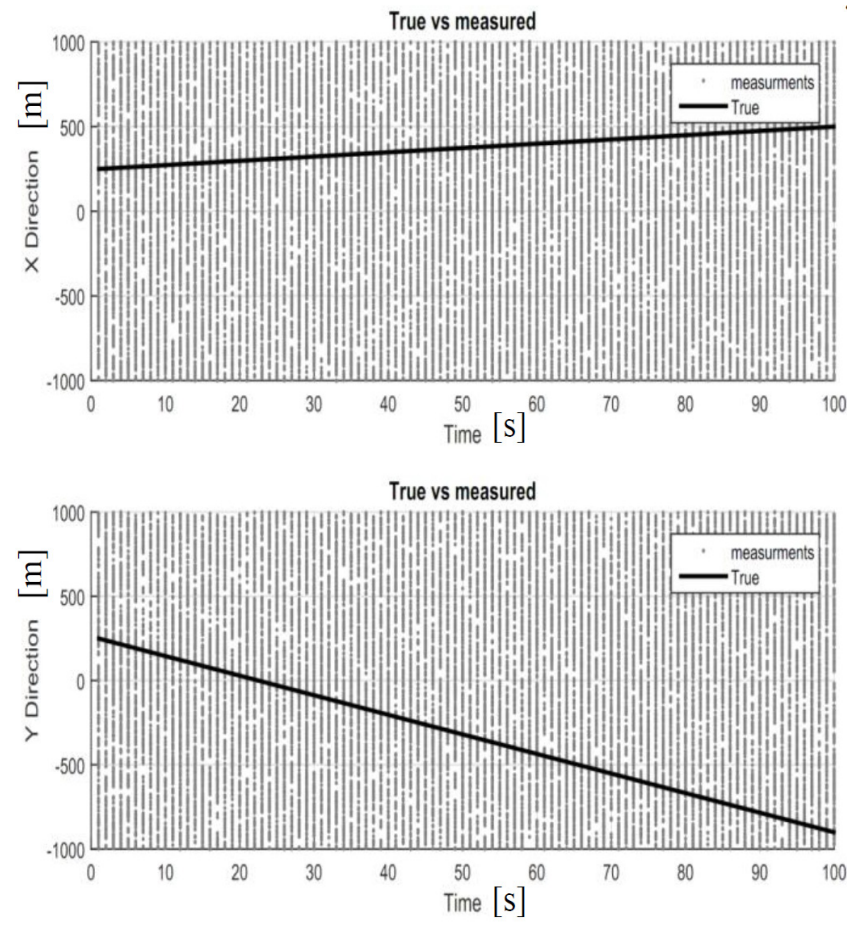

Fig. 6. Observation values of the target and clutter by the sensor along the perpendicular axes $\mathrm{X}$ and $\mathrm{Y}$ separately over time for the single target shown in Fig. 2, N_Clutter $=200$ and after RCMC

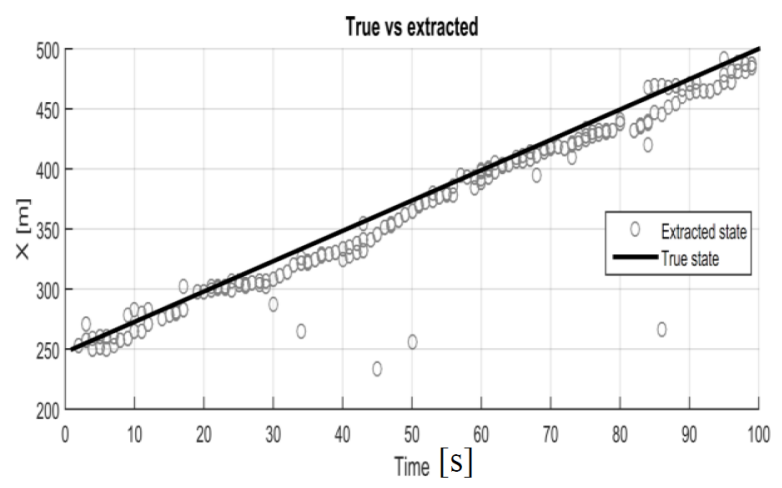

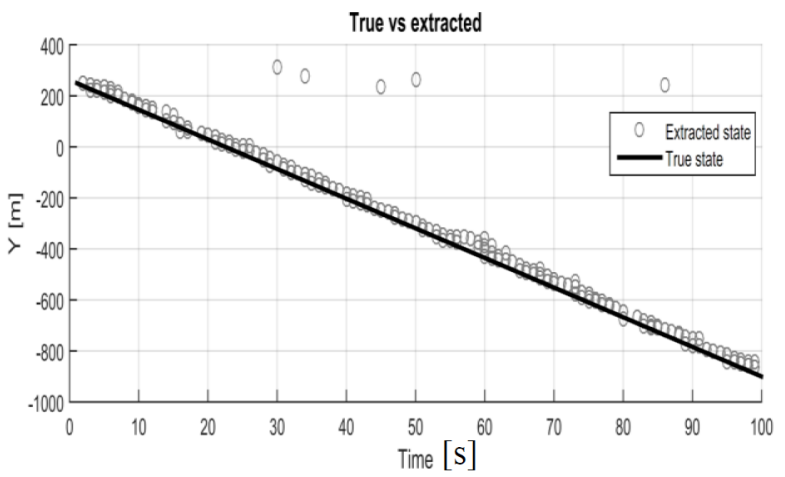

Fig. 7. State estimation of the target using the promoted GM-PHD algorithm along the perpendicular axes $\mathrm{X}$ and $\mathrm{Y}$ separately over time for the single target shown in Fig. 2 and N_Clutter $=200$.

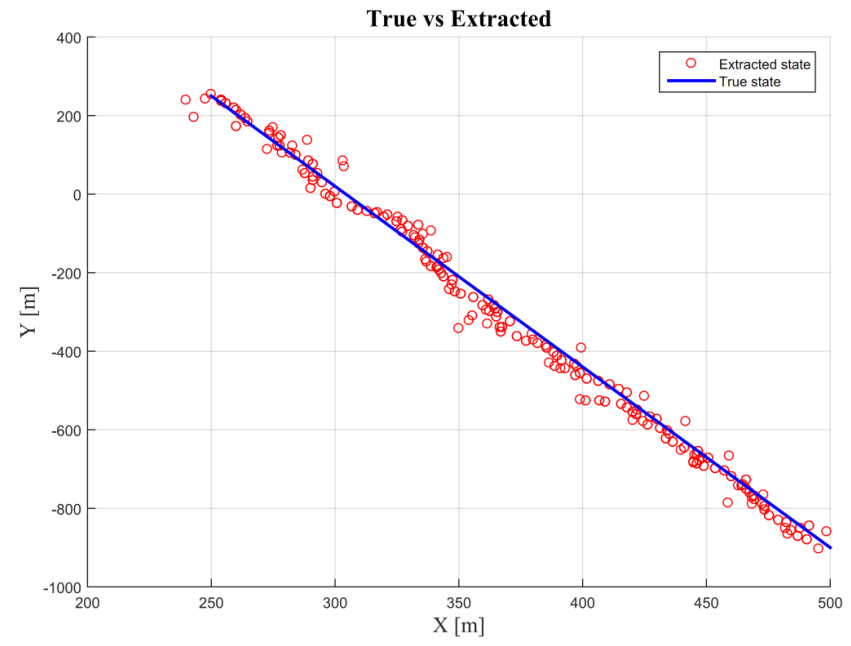

Fig. 8. The 2D target trajectory and the output of the tracking approach by the use of the promoted GM-PHD algorithm for the single target shown in Fig. 2 and $\mathrm{N}$ Clutter $=200$.

In order to compare the performance of the promoted GM-PHD algorithm with ordinary GM-PHD algorithm for tracking of a single moving target in the received raw data by SAR, the tracking results of GM-PHD algorithm are also considered without implementing RCMC. In this case, the observations obtained from sensors (of the target and clutter) are shown in Fig. 9.

The sensor observations of the target with greater density than the clutters with shift-invariance along $\mathrm{X}$ axis (due to radar squint angle) and a curve deviation along $\mathrm{Y}$ axis (due to RCM caused by SAR imaging geometry and motion) are shown in Fig. 9. Due to the faulty observations of sensors regarding the true value in both $\mathrm{X}$ and $\mathrm{Y}$ axis, it is necessary to implement the RCMC process in sensor observations (a radar receiver), before PHD filtering tracking process (or Kalman for single target only) initiates, so as to obtain a true tracking of the moving target in the received raw data by SAR. In fact, Figure 3 is the result of implementation of RCMC process on the observations shown in Fig. 9. Moreover, the estimation results of the state of the moving target shown in previous figures can be obtained and evaluated based on the observations shown in Fig. 9. These results are illustrated in Fig. 10. 


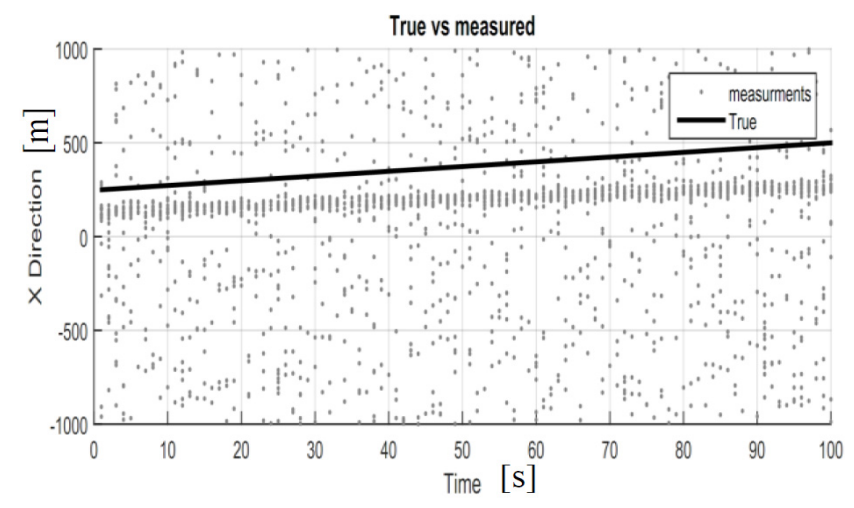

According to Fig. 10, if RCMC compensation process is not implemented in the sensor observations, the estimation of the state of target will be erroneous and the moving target tracking process in the received raw data by SAR material virtually fails. 2-dimensional target trajectory and the result of tracking by ordinary GM-PHD algorithm for this case (if N_Clutter=10) is shown in Fig. 11.

As shown in Fig. 12, the increase in surface density of the clutters (10 times in this sample) results in an increase in the erroneous estimations which shows that the erroneous estimations are caused by clutters not the target(s).

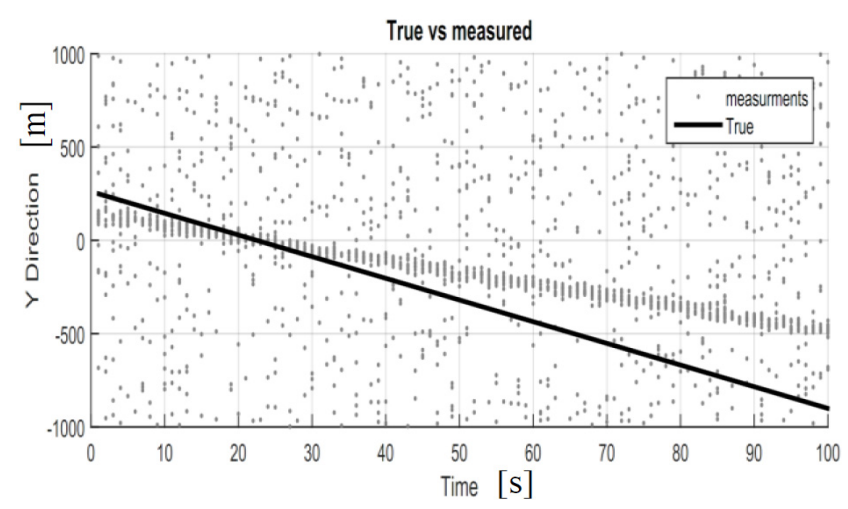

Fig. 9. Observation values of the target and clutter by the sensor along the perpendicular axes $\mathrm{X}$ and $\mathrm{Y}$ separately over time for the single target shown in Fig. 2, N_Clutter $=10$ and without RCMC.
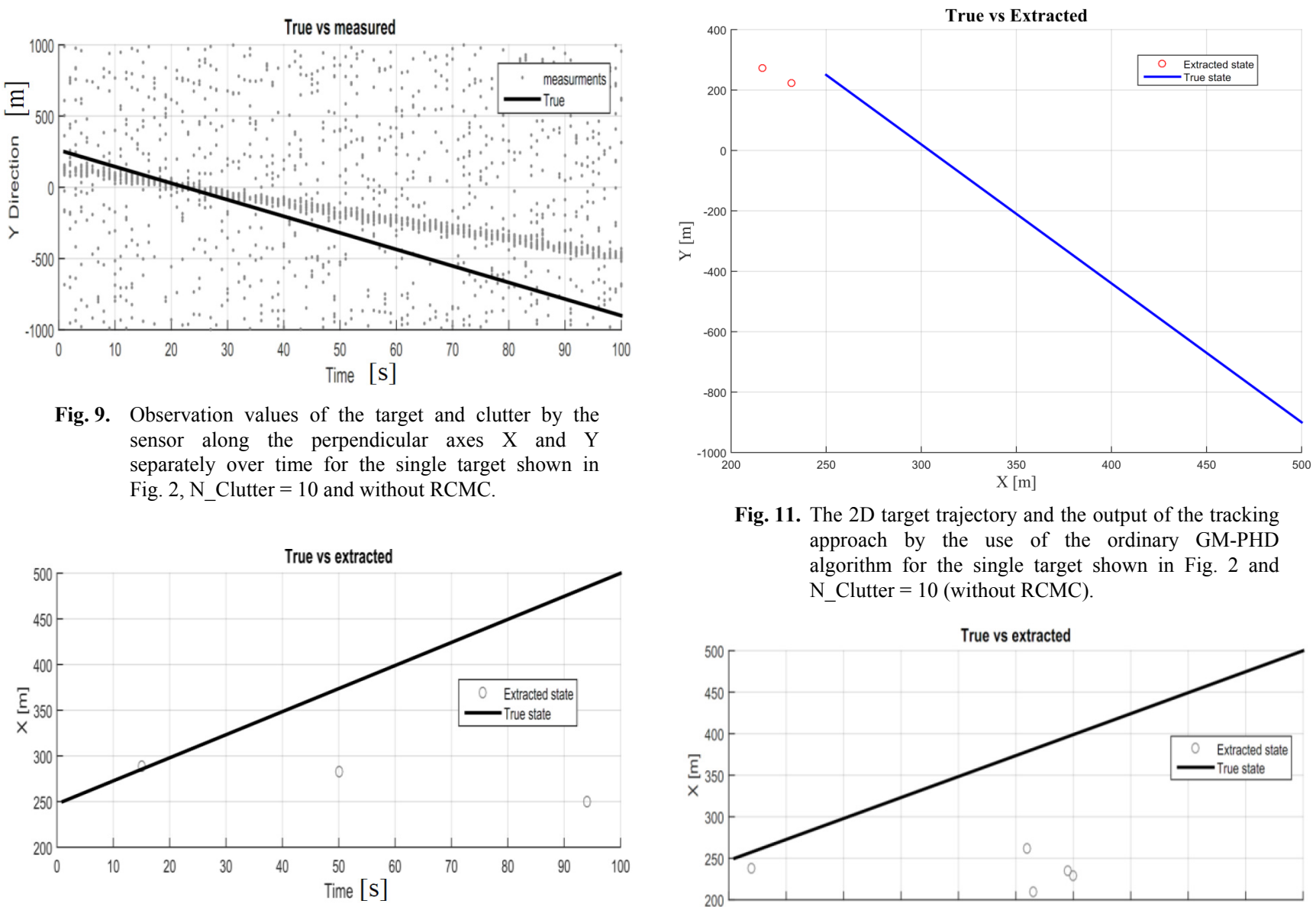

Fig. 11. The 2D target trajectory and the output of the tracking approach by the use of the ordinary GM-PHD algorithm for the single target shown in Fig. 2 and N_Clutter $=10$ (without RCMC).
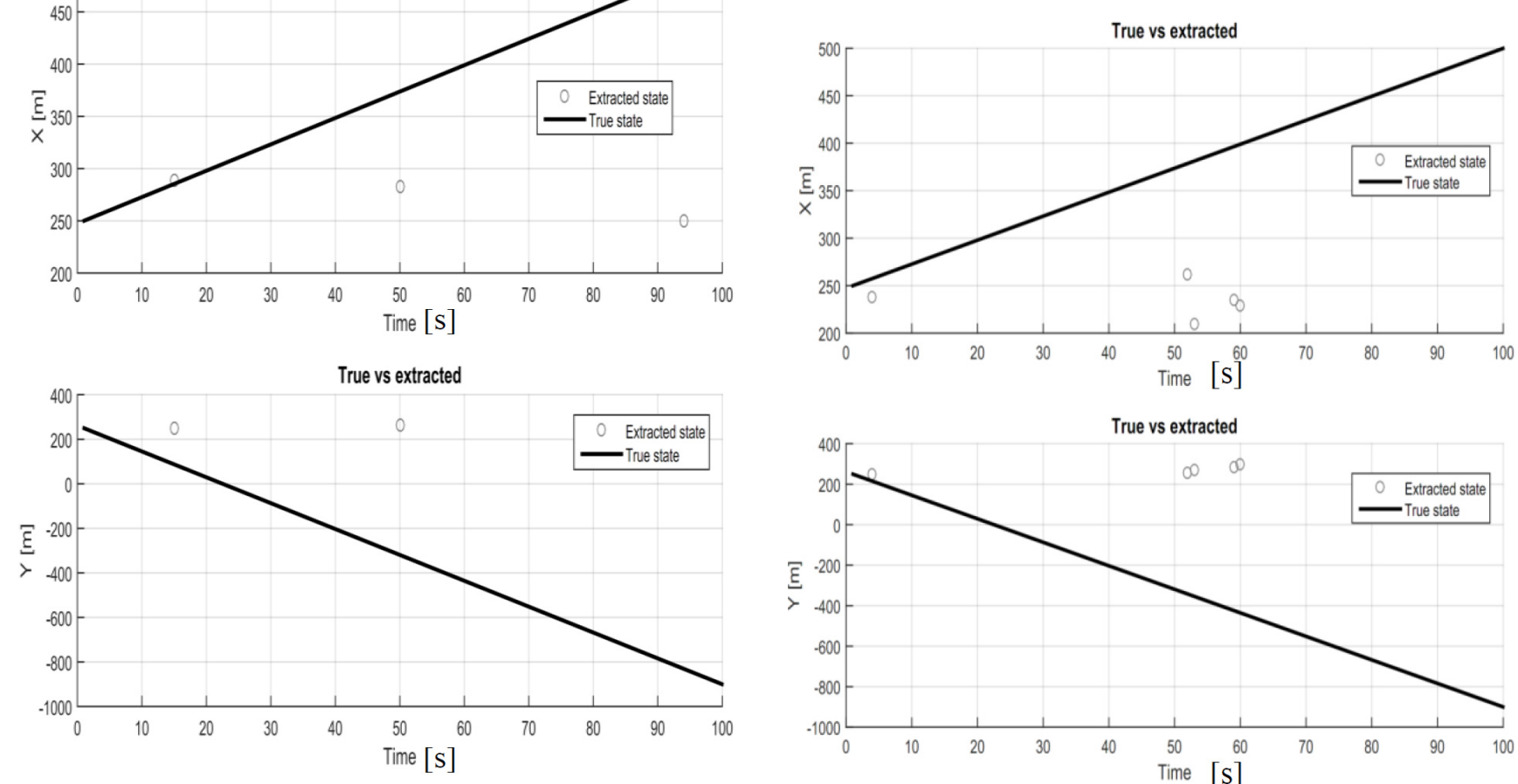

Fig. 10. State estimation of the target using the ordinary GM-PHD algorithm along the perpendicular axes $\mathrm{X}$ and $\mathrm{Y}$ separately over time for the single target shown in Fig. 2 and N_Clutter $=10$ (without RCMC).

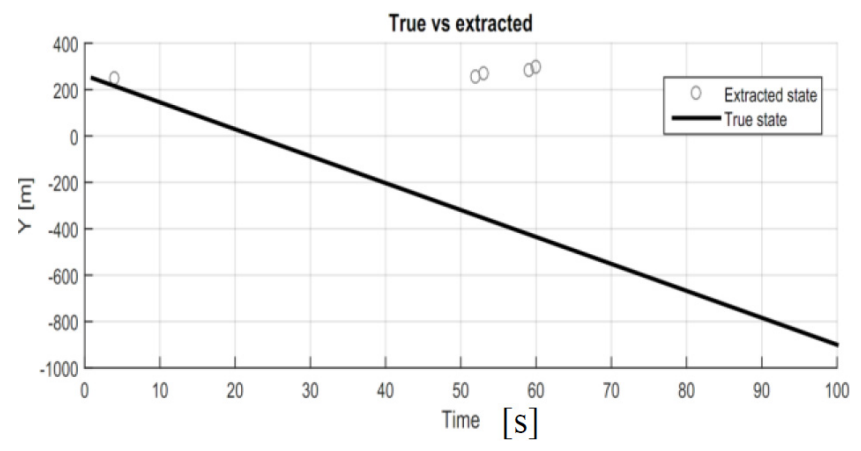

Fig. 12. State estimation of the target using the ordinary GM-PHD algorithm along the perpendicular axes $\mathrm{X}$ and $\mathrm{Y}$ separately over time for the single target shown in Fig. 2 and N_Clutter= 100 (without RCMC). 


\section{Conclusion}

This paper proposed a novel technique based on the GM-PHD filtering for the simultaneous tracking of single moving target in the data received by the SAR in the Spotlight imaging mode. Moreover, the prediction and update equations of the PHD function are in the single-target state space. The implementation of the PHD filter has therefore less computational load than the ideal prediction and update equation in the Bayesian filter. Applying range cell migration compensation on the raw data received by SAR before tracking, made it possible to track single moving target with a good quality.

\section{References}

[1] MAHLER, R. Multi-target Bayes filtering via first-order multitarget moments. IEEE Transactions on Aerospace and Electronic Systems, 2003, vol. 39, no. 4, p. 1152-1178. DOI: 10.1109/TAES.2003.1261119

[2] VO, B.N., SINGH, S. Technical aspects of the probability hypothesis density recursion. Technical Report TR05-006 EEE Department the University of Melbourne, Australia, 2005.

[3] VO, B. N., SINGH, S., DOUCET, A. Sequential Monte Carlo implementation of the PHD filter for multi-target tracking. In Proceedings of the 6th International Conference on Information Fusion. Cairns (Australia), 2003, p. 792-799. DOI: 10.1109/ICIF.2003.177320

[4] VO, B. N., SINGH, S., DOUCET, A. Sequential Monte Carlo methods for Bayesian multi-target filtering with random finite sets. IEEE Transactions on Aerospace and Electronic Systems, 2005, vol. 41, no. 4, p. 1224-1245. DOI: 10.1109/TAES.2005.1561884

[5] VO, B. N., MA, W. K. The Gaussian mixture probability hypothesis density filter. IEEE Transactions on Signal Processing, 2006, vol. 54, no. 11, p. 4091-4104. DOI: 10.1109/TSP.2006.881190

[6] CLARK, D. E., VO, B. N. Convergence analysis of the Gaussian mixture PHD filter. IEEE Transactions on Signal Processing, 2007, vol. 55, no. 4, p. 1204-1211. DOI: 10.1109/TSP.2006.888886

[7] VO, B. N., PASHA, A., TUAN, H. D. A Gaussian mixture PHD filter for nonlinear jump Markov models. In Proceedings of the 45th IEEE Conference on Decision and Control. San Diego (CA, USA), December 13-15, 2006, p. 3162-3167. DOI: 10.1109/CDC.2006.377103

[8] PUNITHAKUMAR, K., KIRUBARAJAN, T., SINHA, A. Multiple model probability hypothesis density filter for tracking maneuvering targets. IEEE Transactions on Aerospace and Electronic Systems, 2008, vol. 44, no. 1, p. 87-98. DOI: 10.1109/TAES.2008.4516991

[9] PASHA, A., VO, B. N., TUAN, H. D, et al. A Gaussian mixture PHD filter for jump Markov system models. IEEE Transactions on Aerospace and Electronic Systems, 2009, vol. 45, no. 3, p. 919 to 936. DOI: 10.1109/TAES.2009.5259174

[10] LI, W. JIA, Y. Gaussian mixture PHD filter for jump Markov models based on best-fitting Gaussian approximation. Signal Processing, 2011, vol. 91, no. 4, p. 1036-1042. DOI: 10.1016/j.sigpro.2010.08.004

[11] NANDAKUMARAN, N., PUNITHAKUMAR, K., KIRUBARAJAN, T. Improved multi-target tracking using probability hypothe- sis density smoothing. In Proceedings of the SPIE Conference on Signal and Data Processing of Small Targets, August 2007, vol. 6699, p. 1-6. ISBN: 9780819468475

[12] NANDAKUMARAN, N., THARMARASA, R., LANG, T., et al. Gaussian mixture probability hypothesis density smoothing with multistatic sonar. In Proceedings of the SPIE Conference on Signal Processing, Sensor Fusion and Target Recognition. March 2008, vol. 6968, p. 1-6. ISBN: 9780819471598

[13] NANDAKUMARAN, N., KIRUBARAJAN, T. Maneuvering target tracking using probability hypothesis density smoothing. In Proceedings of the SPIE Conference on Signal Processing, Sensor Fusion and Target Recognition. March 2009, vol. 7336, p. 1-6. ISBN: 9780763776473

[14] LI, W., JIA, Y., DU, J., et al. Gaussian mixture PHD smoother for jump Markov models in multiple maneuvering targets tracking. In Proceedings of the 2011 American Control Conference. San Francisco (CA, USA), June 29-July 1, 2011, p. 3024-3029. DOI: 10.1109/ACC.2011.5991161

[15] MAHLER, R., VO, B. T., VO, B. N. The forward-backward probability hypothesis density smoother. In Proceedings of the 13th International Conference on Information Fusion. Edinburgh (UK), 2010, p. 1-8. DOI: 10.1109/ICIF.2010.5711920

[16] MAHLER, R., VO, B. T., VO, B. N. Multi-target forwardbackward smoothing with the probability hypothesis density. IEEE Transactions on Aerospace and Electronic Systems, 2012, vol. 48, no. 1, p. 707-728. DOI: 10.1109/TAES.2012.6129665

[17] VO, B. N., VO, B. T., MAHLER, R. A closed form solution to the probability hypothesis density smoother. In Proceedings of the 13th International Conference on Information Fusion. Edinburgh (UK), 2010, p. 1-8. DOI: 10.1109/ICIF.2010.5711983

[18] VO, B. N., VO, B. T., MAHLER, R. Closed form solutions to forward-backward smoothing. IEEE Transactions on Signal Processing, 2012, vol. 60, no. 1, p. 2-17. DOI: 10.1109/TSP.2011.2168519

[19] VO, B. N., SINGH, S., MA, W. K. Tracking multiple speakers with random sets. In Proceedings of the International Conference on Acoustics, Speech and Signal Processing. Montreal (Canada), 2004, vol. II, p. 357-360. DOI: 10.1109/ICASSP.2004.1326268

[20] CHEN, F.L., LIN, H., ZHOU, W., et al. Surface deformation detected by ALOS PALSAR small baseline SAR interferometry over permafrost environment of Beiluhe section, Tibet Plateau, China. Remote Sensing of Environment, 2013, vol. 138, p. 10-18. DOI: $10.1016 /$ j.rse.2013.07.006

[21] YOSHIDA, T., RHEEM, C. K. Time-domain simulation of alongtrack interferometric SAR for moving ocean surfaces. Sensors, 2015, vol. 15 , p. 13644-13659. DOI: $10.3390 / \mathrm{s} 150613644$

[22] JIN, M. J., WU, C. A SAR correlation algorithm which accommodates large range migration. IEEE Transactions on Geoscience and Remote Sensing, November 1984, vol. 22, no. 6, p. 592-597. DOI: 10.1109/TGRS.1984.6499176

[23] CURLANDER, J., MCDONOUGH, R. Synthetic Aperture Radar. New York: John Wiley \& Sons, 1991. ISBN: 978-0-471-85770-9

[24] OLIVER, C. J. Synthetic-aperture radar imaging. Journal of Physics D: Applied Physics, 1989, vol. 25, p. 871-890. DOI: 10.1088/0022-3727/22/7/001

[25] RIEDL, M., POTTER, L., BRYANT, C., et al. Joint synthetic aperture radar and space-time adaptive processing on a single receive channel. IEEE Transactions on Aerospace and Electronic Systems, January 2015, vol. 51, p. 331-341. DOI: 10.1109/TAES.2014.130596

[26] XIA, Y. Synthetic aperture radar interferometry. In Sciences of Geodesy - I. Ed. G. Xu, Springer Berlin Heidelberg, 2010, p. 415 to 474. DOI: $10.1007 / 978-3-642-11741-1$ 
[27] CUMMING, I. G., WONG, F. H. Digital Processing of Synthetic Aperture Radar Data. Norwood: Artech House, 2005. ISBN: 9781580530583

[28] BLACKMAN, S. S. Multiple-target Tracking with Radar Applications. Artech House, 1986. ISBN: 978-0890061794

[29] SMITH, F. W., MALIN, J. A. Models for radar scatterer density in terrain images. IEEE Transactions on Aerospace and Electronic Systems, 1986, vol. 22, no. 5, p. 642-647. DOI: 10.1109/TAES.1986.310730

\section{About the Authors ...}

Navid DARYASAFAR received his B.Sc. and M.Sc. degree in Electrical Telecommunication from Islamic Azad University of Bushehr in 2008 and 2011. Now he is a PhD student of Science \& Research Branch, Islamic Azad University, Tehran. He has been as a senior researcher and lecturer in Islamic Azad University of Bushehr from 2012. His research interests are signal processing, filter design, image processing, acoustic signal, radar recognition, MIMO-OFDM systems.

Ramazanali SADEGHZADEH received the B.Sc. degree in Telecommunication Engineering from K. N. Toosi University of Technology, Tehran, Iran, in 1984, the M.Sc. in Digital Communication Engineering from the University of Bradford, Bradford, UK, and University of Manchester, Institute of Science and Technology (UMIST), Manchester, UK, as a joint program in 1987, and the Ph.D. degree in Electromagnetic and Antenna from the University of Bradford in 1991. During 1992-1997, he worked as a Postdoctoral Research Assistant in the field of propagation, electromagnetic, antenna, biomedical, and wireless communication at the University of Bradford. From 1984 to 1985, he was with the Iran Telecommunication Company, Tehran, Iran, working on networking. Since 1997, he has been with the Faculty of Electrical and Computer Engineering, K. N. Toosi University of Technology. He has published more than 120 referable papers in international journals and conferences. His research interests include numerical techniques in electromagnetics, antenna, propagation, radio networks, wireless communications, nano antennas, and radar systems.

Mohammad NASER-MOGHADASI was born in Saveh, Iran, in 1959. He received the B.Sc. degree in Communication Engineering in 1985 from Leeds Metropolitan University (formerly Leeds Polytechnic), UK. Between 1985 and 1987 he worked as an RF design engineer for the Gigatech Company in Newcastle Upon Tyne, UK. From 1987 to 1989 , he was awarded a full scholarship by the Leeds Educational Authority to pursue an M. Phil. studying in CAD of microwave circuits. He received his Ph.D. in 1993, from the University of Bradford, UK. He was offered then a 2years Post Doc. to pursue research on Microwave Cooking of Materials at the University of Nottingham, UK. From 1995, he joined Islamic Azad University, at Science \& Research Branch, Iran, where currently he is an Associate Professor and head of Telecommunications group. His main areas of interest in research are microstrip antenna, microwave passive and active circuits, RF MEMS. He is a member of the Institution of Engineering and Technology, MIET, and the Institute of Electronics, Information and Communication Engineers (IEICE). He has so far published over 160 papers in different journals and conferences. 